\title{
VICTIMIZACIÓN POR CRIMEN, PERCEPCIÓN DE SEGURIDAD Y SATISFACCIÓN CON LA VIDA EN COLOMBIA*
}

\author{
William Manjarrés de Ávila \\ William Baca Mejia ${ }^{\mathrm{b}}$
}

* DOI: https://doi.org/10.18601/01245996.v21n41.06. Recepción: 27-062017, modificación final: 01-04-2019, aceptación: 14-05-2019. Sugerencia de citación: Manjarrés de Á., W. y Baca M., W. (2019). Victimización por crimen, percepción de seguridad y satisfacción con la vida en Colombia. Revista de Economia Institucional, 21(41), 133-160.

a Magister en Economía. Profesor, Universidad del Atlántico, Barranquilla, Colombia, [williammanjarres@uniatlantico.edu.co], [https://orcid.org/00000001-8128-4778].

b Doctor en Economía. Profesor, Universidad del Norte, Barranquilla, Colombia y St. Charles College, Missouri, EE.UU., [wbaca@uninorte.edu.co], [https://orcid.org/0000-0003-4079-6069]. 
Victimización por crimen, percepción de seguridad y satisfacción con la vida en Colombia

Resumen Este artículo analiza los efectos del crimen y la percepción de seguridad en la satisfacción con la vida. Se estima un probit ordenado cuyos resultados sugieren que la victimización por crimen disminuye en un $1,1 \%$ la satisfacción con la vida; y que un incremento en la percepción de seguridad en la ciudad y en los barrios, la aumentan en un 7,3\% y un 1,7\% respectivamente. Las variables relacionadas con aspectos gubernamentales y equipamiento del barrio resultan significativas y con signo positivo. El género no juega un rol importante, y la pobreza subjetiva disminuye en un $3,8 \%$ la satisfacción con la vida.

Palabras clave: crimen, victimización, percepción de seguridad, satisfacción con la vida; JEL: I31, K42

\section{Crime victimization, safety perception and satisfaction with life in Colombia}

Abstract This paper analyzes the effects of crime victimization and the perception of safety on life satisfaction. We estimated an ordered probit model, and results suggest that crime victimization decreased by $1,1 \%$ life satisfaction. In addition, an increase in the perception of security for the city and neighborhoods, increase life satisfaction at $7,3 \%$ and $1,7 \%$ respectively. Further, other groups of variables are included; government satisfaction and the infrastructure of the neighborhood. All of them have a positive relationship with life satisfaction. Finally, results exhibit that the gender does not play an important role in satisfaction, and subjective poverty decreased by $3,8 \%$ life satisfaction.

Keywords: Crime, victimization, perception of security, life satisfaction; JEL: I31, K42

Vitimização por crime, percepção de segurança e satisfação com a vida na Colômbia

Resumo Este artigo analisa os efeitos do crime e a percepção de segurança na satisfação com a vida. Estima-se um probit organizado, cujos resultados sugerem que a vitimização do crime diminua a satisfação com a vida em $1,1 \%$; e que um aumento na percepção de segurança na cidade e nos bairros tenha aumentado 7,3\% e 1,7\%, respectivamente. As variáveis relacionadas aos aspectos governamentais e às construções de uso público do bairro são significativas e com sinal positivo. $\mathrm{O}$ gênero não desempenha um papel importante, e a pobreza subjetiva diminui a satisfação com a vida em 3,8\%.

Palavras-chaves: crime, vitimização, percepção de segurança, satisfação com a vida; JEL: I31, K42 
1 crimen tiene numerosos efectos en el bienestar: muertes, costos causados por lesiones y robos, y cambios de comportamiento para evitar los crímenes, como despoblamiento de lugares peligrosos (Slogan y Maxfield, 1981); autoprotección privada (Peña, 2005); actitudes sociales más punitivas (Díaz, Ovalle, Rodríguez y Rodríguez, 2001) y pérdida de cultura ciudadana (Ruiz, 2007a, 2007b). E1 crimen está en los primeros lugares de la preocupación pública, y la mayoría de los informes oficiales prestan especial atención a la delincuencia y la inseguridad (Cohen, 2008).

La percepción de seguridad refleja el temor a la delincuencia, y los economistas la utilizan como proxy del costo de la criminalidad (Moore y Shepherd, 2006). Dicho temor afecta el comportamiento económico, influye en la elección del lugar donde se decide vivir y enviar los hijos a la escuela, y cómo se interactúa con la comunidad, se haya sido o no víctima de un delito (Moore y Shepherd, 2006). La satisfacción con la vida es la percepción de cada persona sobre su situación en la vida, dados sus objetivos, expectativas, valores e intereses, en los que influye su contexto cultural (Pérez-E., 2013). E1 crimen y la percepción de seguridad pueden llevar a un bajo nivel de bienestar subjetivo y a la insatisfacción con la vida y, así, a la ansiedad y la depresión (Zamarrón, 2006). América Latina y el Caribe son las regiones con mayores tasas de criminalidad y muertes por violencia (Soares y Naritomi, 2010), y Colombia el país con mayores costos sociales causados por la violencia, en gran parte asociada al narcotráfico, de marihuana en los años setenta y de cocaína en los ochenta (Díaz et al., 2007).

En este artículo se analizan los efectos de la victimización por crimen y la percepción de seguridad en la calidad de vida, medida por la percepción subjetiva, con datos de ocho ciudades colombianas -Barranquilla, Bogotá, Cali, Cartagena, Ibagué, Manizales, Medellín y Valledupar- de 2012 a 2014. Combina datos de delincuencia por ciudades, medidas subjetivas de seguridad en el barrio y la ciudad, y valoraciones subjetivas de satisfacción con la vida. En la primera parte se presenta el marco teórico. En la segunda se revisa la literatura y en la tercera se describen los datos y la metodología. En la cuarta se presentan los principales resultados y en la última, las conclusiones y comentarios finales. 


\section{MARCOTEÓRICO}

\section{SATISFACCIÓN CON LA VIDA}

Desde comienzos de los años setenta, la satisfacción con la vida es tema de investigación debido a la transformación del concepto de calidad de vida, que junto a las condiciones mínimas necesarias para una buena vida (alimentación, vivienda, vestido y salud) incluyó el ambiente psicosocial. Así, la satisfacción con la vida se asocia al sentimiento individual de bienestar o de felicidad. A un alto bienestar subjetivo se lo denomina "felicidad". Una persona tiene un alto bienestar subjetivo cuando está satisfecha con su vida y la asume de manera positiva, es decir, enfrenta las situaciones diarias con optimismo, tranquilidad, sentido del humor y usando sus fortalezas personales (Zamarrón, 2006).

La satisfacción con la vida refleja una valoración de la vida personal a partir de la comparación con una serie de aspectos o dimensiones importantes en la vida (Diener, Suh, Lucas y Smith, 1999; Rojas, 2006). Esta valoración es una medida cognitiva que hace referencia a un juicio global sobre la experiencia vital (Diener, Emmons, Larsen, y Griffin, 1985). Anguas (2000, 2001) identificó varios componentes del bienestar subjetivo, entre ellos el componente cognitivo, al que denomina satisfacción con la vida pues hace referencia a un proceso de valoración de la vida personal en el que se considera un conjunto único de criterios (Pavot, Diener, Colvin y Sandvik, 1991). No obstante, en tales valoraciones pueden influir sesgos de memoria y el estado de ánimo (Kahneman, 1989; Schwarz y Strack, 1999).

Las valoraciones o medidas cognitivas de la satisfacción con la vida se agrupan para establecer una escala de satisfacción con la vida (SWLS, por sus siglas en inglés; Diener, Emmons, Larsen y Griffin, 1985), la medida más citada en la literatura, en la cual las personas indican su nivel de acuerdo o desacuerdo, en una escala de 5 puntos, con cinco afirmaciones que sintetizan su valoración ${ }^{1}$. Se ha demostrado que el uso de las valoraciones personales, aunque sean afectadas por sesgos cognitivos, estados de ánimo y los contextos, son una aproximación válida para medir la satisfacción con la vida (Diener et al., 2012; Schimmack y Oishi, 2005).

${ }^{1}$ Ver [http://www.uv.es/uipd/cuestionarios/accesolibre/ESV.pdf]. Pavot y Diener (2008) y Diener y González (2011) resumen de manera muy completa las maneras de medir la satisfacción con la vida a través de valoraciones personales. 


\section{PERCEPCIÓN DE SEGURIDAD}

La literatura menciona elementos que inciden en la inseguridad y el temor al crimen: victimización, incivilidad, vulnerabilidad física y social y redes sociales. Las explicaciones que se basan en ellos están relacionadas y sirven de base para construir indicadores de percepción de seguridad (Vilalta, 2011; Bissler, 2003).

Para Garofalo (1973, 1979), la victimización es la mayor inseguridad o temor al crimen que sienten personas que han sufrido un delito frente a las que no ha sufrido esa experiencia. Lavrakas y Lewis (1980) añaden que hay diferencias en el tipo de victimización: directa e indirecta. La victimización directa ocurre cuando la persona de referencia es la que ha sufrido algún delito y la indirecta, dicha persona ha sufrido por la victimización de alguien más, bien sea un familiar o un conocido.

La incivilidad consiste en que además de la victimización, hay factores externos que inciden en la experiencia personal y aumentan el temor al crimen (Hunter, 1978). Así, en las zonas donde hay signos de desorden social o deterioro físico son mayores los niveles de percepción de inseguridad. Según este autor, el desorden social termina generando incivilidad y espacios propicios para actos delictivos.

La vulnerabilidad física implica que ciertos grupos - mujeres, adultos mayores, inmigrantes y personas de bajo estrato social- tienden a sentirse más inseguros porque son o se sienten menos capaces de defenderse físicamente en casos de delitos que involucran daño físico (Riger, 1978; Visser et al., 2013). La vulnerabilidad no se limita al aspecto físico, también se presenta a nivel social. La idea de que ciertos grupos son socialmente más vulnerables nació junto a la de vulnerabilidad física y denota la incapacidad de ciertos grupos para prevenir el crimen o recuperarse de los daños producidos (Skogan y Maxfield, 1981). El capital social refleja el nivel de cohesión y cooperación en la comunidad, y las redes sociales son determinantes pues las comunidades con mayor cohesión tienen mayor capacidad para responder colectivamente ante el crimen, y así mejoran la percepción de seguridad (Bissler, 2003).

En un principio, la evidencia criminológica indicó que el temor al delito era un resultado inmediato de la victimización (Amaya, Espinosa y Vozmediano, 2011), entendida como "el acto en el cual una persona es objeto del uso de la fuerza, que le produce un daño físico o psicológico" (Cruz, 1999, p. 260). Hanson, Sawyer, Begle y Hubel (2010) encuentran que haber sido víctima de varios delitos disminuye las habilidades parentales, altera las relaciones íntimas, aumenta los 
conflictos interpersonales, dificulta la consecución y conservación de empleos y las actividades sociales. También tiene consecuencias como el estrés psicológico y desórdenes postraumáticos (Denkers y Winkel, 1998; Russo y Roccato, 2010). Por su parte, Föhrig (2006) argumenta que el temor al crimen puede llegar a ser tan importante como el mismo crimen y Koskela (2011), que el temor al crimen es un grave problema social. No obstante, Kessler (2009) plantea que la sensación de inseguridad no tiene relación con el delito y que es relativamente autónoma, pues a veces el temor aumenta cuando disminuyen las tasas de delitos. Vozmediano, San Juan y Vergara (2008) consideran que el temor al delito es una emoción subjetiva y no tiene una relación directa con estadísticas o índices y objetivos de seguridad y crimen.

Estos distintos puntos de vista indican que es necesario ir más allá de la perspectiva criminológica, y enfocar el fenómeno como parte de un tema más amplio -la percepción de seguridad- que incluye aspectos ambientales, laborales y de participación ciudadana, entre otros (Ruiz, 2007). La medición de la percepción de seguridad es muy importante en los estudios sobre calidad y satisfacción con la vida (San Juan, Vergara y Guzmán, 2005), pues está estrechamente asociada al malestar ciudadano en muchos países.

\section{VICTIMIZACIÓN POR CRIMEN, PERCEPCIÓN DE SEGURIDAD Y SATISFACCIÓN CON LA VIDA}

Algunos economistas analizan los conceptos de calidad de vida, felicidad y bienestar para determinar su relación con diversas variables sociales y económicas (Easterlin, 1974 y 2001; Blanchflower y Oswald, 2004; Clark y Oswald, 1994; Graham y Pettinato, 2002 y Layard, 2005). Esta sección presenta los resultados de trabajos que relacionan la victimización por crimen y la percepción de seguridad con la satisfacción con la vida, considerada como una medida de "bien-estar personal" o de felicidad (Fierro, 2000); la cual se suele basar en la encuestas de percepción que sirven para construir la SWLS ya mencionada.

\section{NORTEAMÉRICA}

La victimización por crimen y la percepción de seguridad se relacionan negativamente con la satisfacción con la vida de diversas maneras. Por ejemplo, el temor al crimen se correlaciona de manera negativa con el bienestar mental (Cornaglia et al., 2014; Lorenc et al., 2012; Stafford et al., 2007). Otros estudios encuentran una relación negativa 
entre victimización por crimen y percepción de la salud (Britt, 2001; Koss et al., 1991; Koss et al., 1990), y entre victimización criminal y seguridad en los barrios (Ward et al., 1986).

Di Tella et al. (2008), con datos de la encuesta Gallup WorldPoll, que se realiza en unos 130 países, encontraron que las víctimas de asaltos y delitos contra el patrimonio tienen menores niveles de bienestar. Estos autores determinan el bienestar subjetivo de cada individuo a través de las preguntas acerca de si los encuestados sonrieron el día anterior a la encuesta o si querrían tener más días como el anterior: La respuestas se emplearon en modelos probabilísticos que muestran los efectos del crimen en las emociones positivas y el bienestar.

Sirgy y Cornwell (2002) revisaron la literatura y analizaron la relación entre satisfacción con la vida y características de los barrios, a las que clasificaron en tres grupos -físicas, sociales y económicas- para ver sus efectos en la calidad de vida. Concluyeron que la satisfacción con características sociales como la satisfacción con la vivienda y el hogar contribuye significativamente al sentimiento de satisfacción con la comunidad en general. Este sentimiento juega un papel importante en la satisfacción con la vida.

Ross y Jang (2000) llegan a resultados similares. Examinan una muestra representativa de 2.482 residentes de Illinois encuestados por teléfono en 1995. Los modelos probabilísticos ordenados que utilizaron indican que las personas que viven en barrios percibidos como desordenados sienten más temor y desconfianza que las que perciben el barrio en orden. La preocupación y el estrés de vivir en un lugar con calles sucias y peligrosas reducen el bienestar. Latkin y Curry (2003) encuentran una relación fuerte positiva entre las características percibidas del barrio y síntomas depresivos posteriores. Sus resultados también sugieren que el barrio y la desorganización social son determinante del estrés crónico.

Algunos estudios muestran que el desorden percibido en los barrios está relacionado con la percepción del bienestar. En su estudio de los barrios de Baltimore, Franzini et al. (2008) concluyeron que la percepción de falta de orden social está relacionada con aspectos observados de desorden físico -fachadas sucias, espacios públicos llenos de basura y graffitis- y social: desventajas económicas y violencia. Latkin et al. (2009) asocian la percepción del barrio a factores objetivos medidos por los informes policiales sobre criminalidad, a experiencias individuales medidas por el tiempo empleado en las calles y a la experiencia de otros medida por la pertenencia a redes. 
Por su parte, Sampson y Raudenbush (2004), con una muestra aleatoria estratificada de 3.585 personas que vivían en Chicago -clasificadas en 478 grupos-, encontraron que la clase y la composición racial predicen el desorden social percibido mejor que el desorden físico. El aumento del porcentaje de personas negras y latinas en cada grupo incrementa la percepción de desorden social; esos resultados fueron significativos al 99\% de confianza para toda la muestra.

Cohen (2008) presenta evidencia del efecto del crimen en la satisfacción con la vida usando una combinación de victimización y datos de la encuesta social de Estados Unidos. Los resultados de sus modelos probabilísticos ordenados indican que las tasas de criminalidad en los condados y la seguridad percibida en los barrios tienen poco impacto en la satisfacción general con la vida del estadounidense promedio; en cambio, un robo en el hogar tiene un efecto considerable. Una posible explicación es que los menores precios de la vivienda y los arriendos más bajos compensan el riesgo de vivir en barrios inseguros.

Michalos y Zumbo (2000) estudian el impacto de la delincuencia en la calidad de vida, la satisfacción con la vida y la felicidad en Prince George, British Columbia. Utilizan como variables explicativas las medidas de temor individual, los datos de victimización, los indicadores de problemas en el barrio y la acción de la policía. Los resultados de sus modelos lineales indican que las víctimas de algún crimen reportan menores niveles de satisfacción con la vida. No obstante, entre los determinantes del bienestar que analizan encuentran que las variables relacionadas con el crimen son compensadas por otras, como la satisfacción con la familia, la salud, y la autoestima.

\section{ÁFrICA}

Sulemana (2015) examina la relación entre victimización criminal y temor al crimen en el bienestar de las personas, usando datos de las encuestas de Afrobarómetro realizadas 20 países de África, y estimando un modelo OLS y un probit ordenado. En ambas estimaciones encuentra que el temor al crimen, la victimización por robo y el asalto físico tienen efectos negativos sobre el bienestar. Además, hace una diferenciación por género y encuentra que mientras el temor al crimen y la victimización por robo se correlacionan significativamente con el bienestar de las mujeres, no afectan el bienestar de los hombres. Sin embargo, el asalto físico reduce significativamente el bienestar de hombres y mujeres.

Davies y Hinks (2010) utilizan datos de 2005 para investigar la relación entre el crimen y la felicidad en Malawi. Su análisis por 
género mostró que hombres y mujeres responden de modo diferente a distintos tipos de crimen. Para los hombres, ser atacados tiene un efecto negativo sobre la felicidad. Para las mujeres, la sensación de inseguridad reduce la felicidad.

Powdthavee (2005) estudió si en la Suráfrica post-apartheid las víctimas de crímenes tenían menor nivel de bienestar subjetivo. Aplicando la encuesta de hogares de octubre de 1997, encontró diferencias significativas entre víctimas de crímenes y no víctimas, con un efecto mayor en los hombres que en las mujeres. Y que la felicidad es menor entre víctimas del crimen, aunque la relación negativa no es tan fuerte cuando la victimización del grupo de referencia es alta. Moller (2005) también encuentra que la victimización por crimen y el temor al crimen reducen la felicidad.

\section{EUROPA}

Hanslmaier (2013) evalúa los efectos de la victimización y la tasa de criminalidad en el temor al crimen y la satisfacción con la vida. Extiende el modelo clásico incorporando los medios de comunicación. Con base en una encuesta nacional realizada en Alemania en 2010 encuentra que el temor al crimen y la victimización disminuyen la satisfacción con la vida. La tasa de criminalidad del condado no tiene un impacto significativo, pero el aumento de las tasas de delincuencia local incrementa el temor al crimen. Esta relación está mediada por los periódicos locales. Los lectores de los diarios se ven más afectados pues tienen más información sobre las tendencias delictivas en su condado.

Staubli et al. (2014) muestran que la delincuencia afecta a la felicidad, usando datos de Swiss Crime Survey 2011. Un robo o intento de robo y el fraude al consumidor, así como los delitos contra la persona, tienen efectos negativos en la satisfacción con la vida. En contra de lo esperado, los efectos perjudiciales sobre la calidad de vida no disminuyen continuamente con el tiempo. Denkers y Winkel (1998), Lelkes (2006), Moore (2006) también encuentran efectos negativos de la victimización por crimen y la percepción de inseguridad en la satisfacción con la vida.

\section{AsIA}

En Asia solo existen dos estudios sobre la relación entre victimización por crimen y la felicidad y calidad de vida. Cheng, Z., y Smyth, R. (2015), examinan la relación entre felicidad, victimización por crimen y seguridad en los barrios de China; utilizan dos modelos 
probit ordenados y encuentran que ser víctima de un crimen o tener un conocido que ha sido víctima tiene un efecto negativo sobre la felicidad, y que vivir en un barrio seguro tiene un efecto positivo. Kuroki (2013) analiza el efecto sobre el bienestar individual por haber sido víctima de robo y hurto. Con microdatos sobre la felicidad en Japón, encuentra una reducción significativa de la felicidad en las personas que han sido víctimas de delitos contra la propiedad.

\section{SURAMÉRICA}

En Colombia, Romero (2014) encuentra que las tasas de criminalidad en Bogotá, en especial la tasa de homicidios, tienen una correlación positiva con la insatisfacción con la vida. Este efecto parece estar mediado por la percepción general de inseguridad y no por la victimización. De modo que no solo es necesario reducir los índices de criminalidad sino también generar una buena percepción de seguridad. Medina y Tamayo (2012) evalúan el efecto de la tasa de homicidios, la percepción de seguridad y la victimización sobre la satisfacción con la vida en Medellín y Bogotá. Encuentran que la tasa de homicidios tiene un efecto negativo y la percepción de seguridad encuentran un efecto positivo, en toda la muestra. Además, que haber sido víctima de un delito tiene un fuerte efecto negativo.

Gaviria et al. (2010) utilizan un modelo de precios hedónicos para estimar el costo del crimen con base en la capitalización del precio de las viviendas de Bogotá. Parten de la hipótesis de que para evitar el crimen, las personas están dispuestas a pagar una "prima de seguridad" sobre el precio de la vivienda. Encuentran que los estratos más altos están dispuestos a pagar hasta un 7,2\% del valor de sus viviendas para evitar un incremento de una desviación estándar sobre la tasa promedio de homicidios. Esto indica que la seguridad, pese a ser un bien público, se distribuye de manera imperfecta en los barrios y se convierte en un bien privado sometido a subasta.

Por último, Medina et al. (2010) estiman modelos de satisfacción con la vida con datos de Bogotá y Medellín, y no encuentran una relación significativa entre crimen y satisfacción con la vida. Consideran que una causa posible es la endogeneidad entre las variables, y no se dispone de datos para corregirla.

\section{OTRAS PERSPECTIVAS}

Tittle y Villemez (1977), examinan el efecto de la clase social en la criminalidad reportada con datos de la gran encuesta realizada en 
1972 a personas mayores de edad en New Jersey, Iowa y Oregón. Los resultados contradicen el planteamiento teórico más común según el cual hay una relación inversa entre clase social y criminalidad. Concluyen que los resultados invitan a replantear esta visión teórica.

Algo después Tittle et al. (1978) analizaron la evidencia empírica sobre la relación entre clase social y criminalidad. Revisaron 35 trabajos y encontraron que solo hay una ligera relación negativa entre las diferencias de clase social y criminalidad. Además, que los trabajos que usan medidas subjetivas de criminalidad reportan una relación menor que los estudios que utilizan estadísticas oficiales. Y plantean serias dudas sobre la idoneidad de las teorías de la criminalidad que incluyen supuestos sobre las diferencias de clase social.

Por otro lado, Blau y Blau (1982) contrastan la hipótesis de que las tasas de violencia criminal urbana son, en gran parte, resultado de las diferencias raciales y la desigualdad socioeconómica. Usan datos de 125 grandes áreas metropolitanas de Estados Unidos y encuentran tres correlaciones importantes con la violencia criminal. Primera, una relación positiva con la ubicación en el sur del país. Segunda, una relación positiva con la proporción de personas negras en la población. Tercera, una relación positiva con la pobreza. Su análisis indica que la desigualdad socioeconómica entre razas, y la desigualdad económica en general, aumentan los índices de violencia criminal, pero una vez se controlan las variables de desigualdad económica los resultados cambian: ni la pobreza ni la ubicación en el sur del país influyen en la violencia criminal, y casi nada la proporción de personas negras. Concluyen que si hay una cultura de la violencia su raíz es la pronunciada desigualdad económica.

Por su parte, Messner (1982) utiliza una muestra de 204 áreas metropolitanas de Estados Unidos para analizar la relación entre pobreza, desigualdad y tasa de homicidios. Sus resultados muestran que la desigualdad de los ingresos familiares está débilmente relacionada con la tasa de homicidios y que el efecto no es significativo. Además, obtiene un resultado inesperado: una relación negativa y significativa entre tasa de homicidios y población en condiciones de pobreza. El autor concluye que es necesario revisar el papel de los factores económicos en la explicación de la tasa de homicidios. Messner y Tardiff (1986) analizan la relación entre niveles de desigualdad económica y tasas de homicidio para una muestra de 26 barrios de Manhattan. Parten de la hipótesis de que un alto grado de desigualdad económica genera altos niveles de privación relativa y altas tasas de homicidio. Sus resultados no apoyan esa hipótesis: la desigualdad económica se 
asocia débilmente con las tasas observadas de homicidios. Así mismo, la composición racial de los barrios no tiene una relación significativa con las tasas de homicidios. En cambio, la proporción de persona en condiciones de pobreza y de personas divorciadas o separadas tiene un efecto fuerte y significativo.

Teniendo en cuenta la revisión de la literatura, este trabajo analiza los efectos de la victimización por crimen y de la percepción de seguridad sobre la satisfacción con la vida, medidas por la percepción subjetiva de las personas encuestadas en las ocho ciudades antes mencionadas, controlando por variables asociadas a la satisfacción con el gobierno, el equipamiento de los barrio y algunos aspectos sociodemográficos de los encuestados.

\section{METODOLOGÍAY DATOS}

Se usan datos de la Encuesta de Percepción Ciudadana: Red de Ciudades Cómo Vamos, que se realiza en 14 ciudades colombianas, de los años 2012, 2013 y 2014. Debido a la discontinuidad de la información se descartaron seis ciudades y el tamaño de muestra resultante fue 37.067 observaciones. Se estima la ecuación:

Satisfacción $_{i}=\beta_{0}+\beta_{\mathrm{j}} \mathrm{S} \& \mathrm{~V}+\beta_{\mathrm{k}} \mathrm{SG}+\beta_{\mathrm{m}} \mathrm{EB}+\beta_{\mathrm{n}} \mathrm{SOC}+\varepsilon_{\mathrm{i}}$

donde la satisfacción con la vida se mide en una escala de 1 a 5 , de Muy insatisfecho a Muy satisfecho teniendo en cuenta las condiciones presentes en cada ciudad para vivir. En el análisis de variables ordinales (un tipo de variables categóricas) se suelen usar regresiones logísticas. Pero esto no es posible porque el modelo logit no puede incluir una variable dependiente con más de dos resultados categóricos ordenados de manera adecuada, y su transformación en una binaria conduciría a una pérdida de información sobre la variable dependiente latente: Satisfacción (Min, 2013).

Otro método usual es el de mínimos cuadrados ordinarios (MCO), en el que la variable dependiente de interés se mide en una escala continua. Si se viola este supuesto (como cuando es categórica), surgen graves problemas:

1. Predicciones fuera del rango de la escala de la variable dependiente categórica nominal.

2. Pruebas de hipótesis no válidas de los coeficientes de las variables explicativas (valores $\mathrm{t}$ ) y de la curva de regresión (valores $\mathrm{F}$ ).

3. Como consecuencia de (1) y (2i), el R $\mathrm{R}^{2}$ como medida de la bondad del ajuste puede ser engañoso (Moutinho, Peel y Goode, 1998). 
Por ello, Long y Freese (2006) argumentan que el modelo con mejor ajuste y más adecuado para manejar variables ordinales es el probit ordenado. También existen modelos logit ordenados que utilizan una función de distribución acumulativa de la distribución logística, y modelos probit que utilizan la función de distribución acumulativa de la distribución normal. Ambos métodos producen inferencias similares pero no idénticas (Moscote y Rincón, 2012).

Para seleccionar el modelo, un logit o un probit ordenado, se tiene en cuenta el criterio de información de Akaike AIC (Akaike, 1974), que maximiza la log-verosimilitud esperada empleando máxima verosimilitud, una aplicación del principio de maximización de la entropía (Kullback y Leibler, 1951). El criterio AIC refleja la bondad de ajuste, y selecciona el modelo con $k$ factores tal que $\operatorname{AIC}(k)$ alcanza el valor mínimo. De acuerdo con Takane (1987), el AIC no identifica el modelo verdadero. Aunque el modelo seleccionado es el que mejor se ajusta a los datos no es el modelo real o verdadero. Es el mejor entre los candidatos, en el sentido de que más se acerca a la realidad. Con los datos que aquí se utilizan el valor del AIC para el modelo probit ordenado es de 86540,79 y para el logit ordenado de 86599,13 . De modo que se escoge el modelo probit ordenado.

En este modelo se estima una puntuación subyacente como función lineal de las variables independientes y un conjunto de puntos de corte, cinco en este caso. La probabilidad de observar el resultado $i$ es la probabilidad de que la función estimada, más el error aleatorio, esté dentro del rango de los puntos de corte estimados para el resultado: $\operatorname{Pr}\left(\right.$ resultado $\left._{j}=i\right)=\operatorname{Pr}\left(k_{i-1}<\beta_{1} x 1_{j}+\beta_{2} x_{2 j}+\ldots+\beta_{k} x_{k j}+\mu_{j} \leq k_{i}\right)$

Se supone que $\mu_{j}$ se distribuye normalmente. Se estiman los coeficientes $\beta_{1}, \beta_{2}, \beta_{k}$ y los puntos de corte, $k_{1}, k_{2}, \ldots k_{i-1}$, donde $i$ es el número de posibles resultados. Aquí nos centramos en la significancia estadística de los $\beta_{i}$ de las variables explicativas agrupadas según sus características, en cuatro grupos:

- Seguridad y victimización (SESV): conforme a la revisión de la literatura este grupo incluye variables, medidas en la escala de 1 a 5 , asociadas a la sensación de seguridad (percepción de seguridad en la ciudad y en el barrio donde se habita) y un índice de victimización relacionado con haber sido o no víctima de crimen en los últimos doce meses.

- Satisfacción con el gobierno $(S G)$ : este grupo incluye variables de percepción, también medidas en la escala de 1 a 5 , relacionadas con los actores gubernamentales y su gestión en la ciudad (favorabilidad 
del alcalde, satisfacción con la gestión del equipo de gobierno, favorabilidad del concejo, satisfacción con la inversión en bienes públicos). Y, además, variables de cumplimiento de la ley y garantía del derecho a la salud, medidas como una probabilidad de 0 a 1 .

- Equipamiento del barrio (EB): este grupo está compuesto por medidas de satisfacción relacionadas con las condiciones físicas del área geográfica: vías, alumbrado público, parques y zonas verdes, andenes y separadores de calle y servicios públicos domiciliarios. También, se incluye una variable de servicios públicos, un índice compuesto con valores de 0 a 1.

- Socioeconómicas $(S O C)$ : este grupo de control incluye variables de características individuales. La encuesta permite usar la edad por categorías: 1, de 18 a 25 años; 2, 26 a 35 años; 3, de 36 a 45 años; 4, de 46 a 55 años; y 5, más de 55 años. Además, se incluye el género y una medida de pobreza subjetiva, en una escala de 0 a 1 , donde 1 se considera pobre y 0 en otro caso, para mejorar la eficiencia de los estimadores.

En materia de cumplimiento de la ley ${ }^{2}$ y satisfacción con los servicios públicos ${ }^{3}$, la encuesta hace preguntas sobre cada componente; se construyeron índices compuestos que captan su multi-dimensionalidad con el método de componentes principales (ACP), que explica la mayor parte de la variabilidad observada en un conjunto de variables con el menor número posible de componentes (Jiménez, 1985). Este método transforma linealmente el conjunto de variables originales, que suelen estar correlacionadas, en un conjunto de variables no correlacionadas -los componentes principales- ordenadas por el porcentaje de variabilidad que explican. Luego se escogen los componentes que explican la mayor variabilidad acumulada; así se reduce el tamaño del conjunto de información. Los componentes así obtenidos no constituyen un indicador o índice per se. Para obtener índices a partir de ellos se usa el método propuesto por Giovannini,

\footnotetext{
${ }^{2}$ Las variables que componen este índice corresponden a la probabilidad de ser castigado o amonestado por las autoridades si se violan normas de construcción y urbanismo; se arroja basura a la calle; se daña un bien público; se paga para evadir trámites; se conecta ilegalmente a servicios públicos; se invaden espacios públicos; se incumplen normas ambientales; no se pagan impuestos; se orina en el espacio público; se incumplen normas de tránsito; se portan armas; y se agrede a otra persona.

${ }^{3}$ Las variables que componen este índice corresponden a la satisfacción con la prestación de servicios de acueducto, alcantarillado, energía eléctrica, telefonía celular, aseo y recolección de basuras, gas domiciliario e internet.
} 
et al. (2005), de modo que el ACP ayuda a calcular las ponderaciones; y luego se re-escala la agrupación.

Al reducir la dimensionalidad del sistema se pierde información. Para probar que la pérdida no es grande, se comprueba la fiabilidad de las variables que se incluirán en los índices con el coeficiente a de Cronbach (1951), que toma valores de 0 a 1 y sirve para comprobar si un indicador recopila información de las variables que lo componen; cuanto más se acerca a 1 , mayor es la fiabilidad de la variables escogidas; indica una buena fiabilidad a partir de 0,70 (Schuschny y Soto, 2009). En este trabajo el a de los componentes del índice de cumplimiento de la ley es de 0,93 y el de satisfacción con los servicios públicos es de 0,81 .

\section{Pruebas de RobusteZ}

Powdthavee (2005) señala que la causalidad entre la victimización y las variables de felicidad y satisfacción con la vida no es del todo clara. Aunque se considera que la victimización por crimen es exógena, pues suele ocurrir al azar, los estudios de criminología y victimología sugieren que el riesgo de victimización puede depender de características personales, ambientales y socio-económicas. De acuerdo con las nociones de actividad rutinaria y exposición por el estilo de vida, la victimización por crimen puede ocurrir debido a que estos elementos pueden poner a algunas personas o a su propiedad en las proximidades de delincuentes (Cohen y Felson, 1979). En otros términos, no solo se debe tener en cuenta el impacto de los lugares en las personas, sino también el impacto de las personas en los lugares (Propper et al., 2007). Esto implica que hay un problema de autoselección, que puede sesgar las estimaciones paramétricas y poner en duda la relación causal entre victimización y satisfacción con la vida. Debido a la posible endogeneidad de la victimización, se debe buscar una manera de asegurar la significancia del efecto que se estima con el modelo probit ordenado.

Una manera de abordar este problema es hacer un experimento en el que se victimice a las personas al azar; lo que es ilegal y nada ético. Una segunda manera es utilizar datos de panel. Pero esta no es una opción porque en la encuesta solo hay datos de corte transversal. La tercera es usar una variable instrumental, correlacionada con la victimización por crimen pero no con el término de error, es decir, con todo lo que no pudo explicar el modelo estimado. Como muestran Cheng y Smyth (2015), pocos estudios abordan la endogeneidad de la victimización por crimen usando variables instrumentales, sin 
demostrar que satisfacen el criterio de exclusión o exogeneidad, es decir que están correlacionados con la victimización pero no con las características personales, ambientales y socio-económicas. A partir de la encuesta que aquí se utiliza no se pueden obtener variables instrumentales que satisfagan ese criterio. La última manera, que proponen Cheng y Smyth, es separar el efecto de la victimización del sesgo de autoselección. Esto implica utilizar el emparejamiento por puntaje de propensión (Propensity score matching, PSM) para reducir la dependencia del modelo y hacer inferencias, con base en el supuesto de independencia condicional estándar, según el cual dependiendo del vector de características observadas $X$, la variable de tratamiento es independiente de los resultados potenciales. PSM calcula el efecto promedio del grupo de tratamiento sobre el grupo tratado (ATT), en este caso la diferencia en el resultado promedio (satisfacción) de quienes dicen haber sido víctimas de la delincuencia $(T=1)$ y los que no lo han sido $(\mathrm{T}=0)$ :

$A T T=E[$ Satisfacción $\mid T=1, P(X)]-E[$ Satisfacción $\mid T=0, P(X)]$

$E[\bullet]$ denota la esperanza matemática, $T$ es la variable de tratamiento (haber sido víctima de un delito), y $P(X)$ es la puntuación derivada de las variables observadas en $X$, de modo que la distribución condicional de $X$, dada $P(X)$, sea la misma para los grupos tratados y no tratados.

Dada la naturaleza de los datos de la encuesta se escogió esta última aproximación, PSM. Pero son de corte transversal y es posible que no se puedan controlar los efectos fijos no observables (condiciones individuales) y que haya un sesgo oculto. Aunque PSM controla las variables observadas, el sesgo oculto puede surgir si existen variables no observables que no se pueden controlar en el emparejamiento y que afecten simultáneamente la probabilidad de ser víctima y la satisfacción. Para evitar este problema se evalúa la sensibilidad de los resultados a posibles variables no observadas.

$\mathrm{El}$ análisis de sensibilidad proporciona un aumento cuantificable de la incertidumbre cuando se relaja un supuesto clave. Rosenbaum (2002) propuso un análisis de sensibilidad para determinar si la estimación por emparejamiento es robusta a la posible presencia de un factor de confusión no observado - efectos fijos no observados- que afecta la selección y con ella las implicaciones del PSM.

Según el supuesto del PSM, la probabilidad de ser víctima de crimen debe ser explicada únicamente por las variables en $X$. Si no es así, dos personas con iguales valores en $X$ tendrán diferentes probabilidades de ser víctimas. Rosenbaum define un valor gamma $(\Gamma)$ en 
función de esa probabilidad, es decir, cuando se cumple el supuesto. Valores más altos de $\Gamma$ significan que el estudio difiere cada vez más del contexto experimental. Cuanto mayor es el nivel de $\Gamma$ para el cual el ATT sigue siendo estadísticamente diferente de 0 , mayor es la robustez de las estimaciones ante posibles sesgos ocultos.

\section{DESCRIPCIÓN DE LAS VARIABLES}

E1 cuadro 1 muestra las estadísticas descriptivas de las variables. Las personas encuestadas están relativamente satisfechas con la vida en la ciudad en que habitan. A la pregunta correspondiente respondieron que se sienten satisfechas, con un puntaje promedio de 3,97. Pero este nivel de satisfacción no se complementa con la percepción de seguridad, que en promedio es menor porque se sienten medianamente seguras. E1 16\% reportó que había sido víctima de un delito en el año anterior a la encuesta. Los principales delitos incluyen atraco, robo -a residencia, de vehículo o en transporte público-, extorsión o vacuna, estafa y amenazas.

Cuadro 1

Estadísticas descriptivas

\begin{tabular}{lcc}
\hline Variables & Media & Desviación estándar \\
\hline Satisfacción con la ciudad & 3,97 & 1,01 \\
\hline Seguridad y victimización & & \\
Seguridad en la ciudad & 2,95 & 1,19 \\
Seguridad en el barrio & 3,39 & 1,19 \\
Víctima de crimen & 0,16 & 0,37 \\
\hline Satisfacción con el gobierno & 2,25 & 0,76 \\
\hline Favorabilidad del alcalde & 2,91 & 1,10 \\
Satisfacción con la gestión del equipo de gobierno & 1,93 & 0,82 \\
Favorabilidad del concejo & 2,97 & 1,16 \\
Satisfacción con la inversión de recursos públicos & 0,56 & 0,19 \\
Cumplimiento de la ley & 0,54 & 0,50 \\
Garantía al derecho a la salud & & 1,28 \\
\hline Satisfacción con el equipamiento del barrio & 3,40 & 1,13 \\
\hline Vías & 3,72 & 1,23 \\
Alumbrado público & 3,30 & 0,16 \\
Andenes y separadores de las calles & 0,77 & 1,48 \\
Servicios públicos & & 0,50 \\
Socioeconómicas & 3,25 & 0,42 \\
\hline Edad & 0,49 & 0,23 \\
Género & & \\
Pobreza subjetiva & & \\
\hline
\end{tabular}

Fuente: Encuesta de Percepción Ciudadana:Red de Ciudades Cómo Vamos (2012,2013 y 2014), elaboración propia.

Los resultados indican que no hay grandes diferencias en la proporción de personas víctimas de algún tipo de delito en las ciudades. 
Pero en la percepción de seguridad sí hay diferencias; mientras que en Manizales, Ibagué y Valledupar, un $45 \%$ en promedio se siente seguro o muy seguro, en ciudades como Barranquilla, Bogotá, Cali, Cartagena, y Medellín solo el 31\% en promedio se siente seguro o muy seguro. En Barranquilla y Cali mejoró la percepción de seguridad entre 2012 y 2014, mientras que en Cartagena se mantuvo igual y en Bogotá y Medellín disminuyó. A pesar de la disminución, Medellín sigue siendo la de mejor percepción de seguridad; el 44\% de los encuestados se siente seguro o muy seguro. En Ibagué y Valledupar mejoró la percepción de seguridad y en Manizales tuvo una ligera disminución entre 2012 y 2013.

Una mayor proporción de encuestados se siente más segura en el barrio que en la ciudad, un 10\% más en promedio. Medellín también tiene la mejor percepción de seguridad en el barrio y la mayor diferencia entre ambas percepciones, un 30\% más en 2014. Mientras que Bucaramanga (38\%), Cartagena (33\%) y Valledupar (38\%) tienen los menores porcentajes de encuestados que se sienten seguros en su barrio. Un análisis más detallado indica que así disminuyan los delitos de alto impacto -homicidios, robo a residencias, comercios, etc.- en la percepción de seguridad influyen ante todo los de menor impacto como el atraco callejero. No es necesario ser víctima para que desmejore la percepción de seguridad (Jiménez, 2015). Jiménez señala que intervienen otros factores; basta que a una persona le digan que hubo un crimen en cierto lugar o que presencie un atraco para que la percepción de seguridad desmejore.

También se observó una baja proporción de denuncias. En promedio, cuatro de cada diez víctimas de algún delito lo denunciaron ante las autoridades. Entre las principales razones para no hacer la denuncia se encuentra la desconfianza en las autoridades ${ }^{4}$. La imagen del alcalde tiende a ser desfavorable y peor que la del concejo; en promedio, un mayor número de personas manifestó no saber el nombre del alcalde. La satisfacción con la inversión de recursos públicos en la ciudad se ubica en una escala media-neutral; en 2012, 2013 y 2014 , solo se encontraban satisfechos el $28 \%$, el $29 \%$ y el $31 \%$ de los encuestados, respectivamente. En la satisfacción con las condiciones y los bienes públicos del barrio, los resultados muestran un nivel medio de satisfacción; el alumbrado público obtuvo el mayor valor promedio. En cuanto a los aspectos socioeconómicos, la encuesta fue respondida,

${ }^{4}$ El número de denuncias aumentaría si se mejora la confianza en las autoridades y se crean condiciones para que las personas se sientan seguras cuando denuncian los delitos. 
en una proporción similar, por hombres y mujeres de 36 a 45 años de edad y, en promedio, el 54\% considera que se les respeta su derecho a la salud, un porcentaje que pasó del 50\% en 2012 al 56\% en 2014. Por último, el $23 \%$ se considera pobre, un porcentaje relativamente estable, del 23\% en 2012, del 21\% en 2013 y del 23\% en 2014.

\section{ESTIMACIÓN Y RESULTADOS}

El cuadro 2 presenta los resultados de las estimaciones para toda la muestra. La primera columna muestra que la gran mayoría de variables fueron significativas y con el signo adecuado en todas las agrupaciones. A continuación, se detalla el resultado de cada grupo de variables.

Cuadro 2

Estimaciones

\begin{tabular}{|c|c|c|c|c|c|c|}
\hline Variables & Modelo total & Muy insatisfecho & Insatisfecho & $\begin{array}{l}\text { Ni satisfecho ni } \\
\text { insatisfecho }\end{array}$ & Satisfecho & Muy satisfecho \\
\hline \multicolumn{7}{|l|}{ Seguridad y victimización } \\
\hline Seguridad en la ciudad & $0,2607692^{* * * *}$ & $-0,0131603^{\text {***a* }}$ & $-0,0204843^{\text {*at*ak }}$ & $-0,0391633^{\text {**ak*ak}}$ & $-0,0085487^{\text {*****a* }}$ & $0,0813567^{\text {米:***a }}$ \\
\hline Seguridad en el barrio & 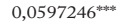 & 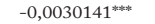 & $-0,0046916^{\text {*Nake }}$ & 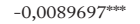 & 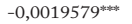 & $0,0186333^{\text {*wak }}$ \\
\hline Víctima de crimen & $-0,0384785^{\text {*ak }}$ & $0,0019419^{\text {*** }}$ & $0,0030226^{* *-k}$ & $0,0057788^{* *}$ & $0,0012614^{* * *}$ & $-0,0120048^{\text {**** }}$ \\
\hline \multicolumn{7}{|l|}{ Satisfacción con el gobierno } \\
\hline Favorabilidad del alcalde & $0,0227428^{* * * * *}$ & $-0,0011478^{* * * *}$ & $-0,0017865^{\text {*****at }}$ & $-0,0034156^{* \text { *atk }}$ & $-0,0007456^{\text {柱水水 }}$ & $0,0070955^{\text {*** }}$ \\
\hline $\begin{array}{l}\text { Satisfacción con la gestión } \\
\text { del equipo de gobierno }\end{array}$ & $0,0431372^{* * * *}$ & $-0,002177^{* * *}$ & $-0,0033886^{* * * * *}$ & 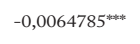 & $-0,0014141^{\text {***:a }}$ & $0,0134583^{\text {*N:e*k }}$ \\
\hline Favorabilidad del concejo & 0,0104303 & $-0,0005264$ & $-0,0008193$ & $-0,0015665^{*}$ & $-0,0003419$ & 0,0032541 \\
\hline $\begin{array}{l}\text { Satisfacción con la inversión } \\
\text { de recursos públicos }\end{array}$ & $0,1196906^{\text {*a:-ak }}$ & 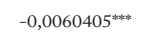 & 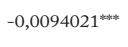 & $-0,0179756^{* * * *}$ & $-0,0039238^{\text {****: }}$ & $0,0373419^{* * * * *}$ \\
\hline Cumplimiento de la ley ${ }^{1}$ & $0,0574474^{\text {*a*k }}$ & $-0,0028992^{* *}$ & $-0,0045127^{* * *}$ & $-0,0086277^{* *}$ & $-0,0018833^{* * *}$ & $0,0179229^{\text {**akk }}$ \\
\hline Garantía del derecho a la salud & $0,0864035^{\text {***** }}$ & $-0,0043606^{* * a-k}$ & 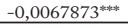 & 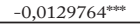 & $-0,0028325^{\text {承水 }}$ & $0,0269568^{\text {**ak }}$ \\
\hline \multicolumn{7}{|c|}{ Satisfacción con el equipamiento del barrio } \\
\hline Vías & $0,0209454^{* * * *}$ & 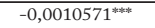 & $-0,0016453^{\text {wowk }}$ & $-0,0031457^{\text {*watek }}$ & $-0,0006866^{\text {talk }}$ & $0,0065347^{\text {米冰水 }}$ \\
\hline Alumbrado público & $0,0532227^{* * * *}$ & $-0,002686^{* * * *}$ & $-0,0041808^{* * * *}$ & $-0,0079932^{* * * * *}$ & $-0,0017448^{* 0 * * * *}$ & $0,0166048^{\text {*kek }}$ \\
\hline Andenes y separadores de calles & 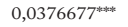 & $-0,001901^{* * * *}$ & $-0,0029589^{* * * *}$ & 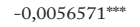 & $-0,0012348^{* w_{*}^{*} \cdot k}$ & 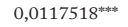 \\
\hline Servicios públicos ${ }^{1}$ & $0,4850314^{* * * * *}$ & $-0,0244783^{\text {**ak }}$ & $-0,0381009^{\text {*a*k }}$ & $-0,0728439^{\text {**ak }}$ & $-0,0159005^{\text {kato: }}$ & $0,1513236^{\text {**** }}$ \\
\hline \multicolumn{7}{|l|}{ Socioeconómicas } \\
\hline Edad & $0,0346873^{\text {***** }}$ & $-0,0017506^{* \text { *ak }}$ & 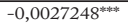 & $-0,0052095^{\text {*akak }}$ & 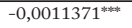 & $0,010822^{* * * * *}$ \\
\hline Género & $-0,0013321$ & 0,0000672 & 0,0001046 & 0,0002001 & 0,0000437 & $-0,0004156$ \\
\hline Pobreza subjetiva & $-0,1360401^{* * * *}$ & $0,0068656^{* * *}$ & $0,0106864^{\text {***** }}$ & $0,020431^{* * * * * *}$ & $0,0044597^{\text {校* }}$ & $-0,0424428^{\text {*wak }}$ \\
\hline \multicolumn{7}{|l|}{ Ciudades (Barranquilla) } \\
\hline Bogotá & $-0,5512718^{\text {***** }}$ & $0,0304576^{* * * *}$ & $0,0479134^{* * * * *}$ & $0,0872154^{* \text { **** }}$ & $0,0037063^{\text {*** }}$ & $-0,1692927^{\text {来准 }}$ \\
\hline Cali & $-0,3199206^{* * * *}$ & $0,0142678^{* * * *}$ & $0,0251516^{* * * * *}$ & $0,0517713^{\text {****** }}$ & $0,0118505^{\text {***** }}$ & $-0,1030411^{\text {*tak }}$ \\
\hline Cartagena & $-0,4011025^{\text {***** }}$ & $0,0192972^{* * * *}$ & $0,0327303^{\text {****** }}$ & $0,064603^{* * * *}$ & $0,0105862^{* * *}$ & $-0,1272167^{\text {*ak }}$ \\
\hline Ibagué & 0,0386674 & $-0,0012297$ & $-0,0025227$ & $-0,0061553$ & $-0,0031763$ & 0,013084 \\
\hline Manizales & $0,0947458^{\text {*kte*k }}$ & $-0,0028585^{\text {k*k**** }}$ & $-0,0059874^{* * * *}$ & $-0,0149686^{* * *}$ & $-0,0084012^{* * * *}$ & $0,0322157^{* * * *}$ \\
\hline Medellín & 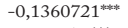 & 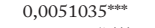 & $0,0097619^{\text {**:**: }}$ & $0,021993^{\text {: }}$ & $0,0082657^{* * * *}$ & $-0,045124^{* * * *}$ \\
\hline Valledupar & $0,142278^{* \text { *atek }}$ & $-0,0041057^{\text {米㸠 }}$ & $-0,0087477^{\text {米桃 }}$ & $-0,0223133^{\text {*at***a }}$ & $-0,0133809^{* * * *}$ & $0,0485476^{* * * *}$ \\
\hline \multicolumn{7}{|l|}{ Años } \\
\hline 2013 & 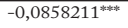 & $0,0042604^{\text {salk:k }}$ & $0,0066845^{\text {*k:k*k }}$ & $0,0128908^{\text {tate:k }}$ & 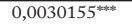 & $-0,0268512^{* * * *}$ \\
\hline 2014 & $-0,0639619^{\text {*k-ak }}$ & $0,0031183^{\text {*ak: }}$ & $0,0049362^{\text {*wak }}$ & $0,009614^{\text {*ank }}$ & $0,0024093^{\text {**:*k }}$ & $-0,0200777^{\text {******}}$ \\
\hline Observaciones & 37.067 & 37.067 & 37.067 & 37.067 & 37.067 & 37.067 \\
\hline Log de verosimilitud & $-43240,39$ & & & & & \\
\hline Pseudo $\mathrm{R}^{2}$ & 0,1120654 & & & & & \\
\hline
\end{tabular}

${ }^{1}$ Índices compuestos. ${ }^{*}: p<0.10,{ }^{* *}: p<0.05,{ }^{* * *}: p<0.01$.

Fuente: elaboración propia.

\section{SEGURIDAD Y VICTIMIZACIÓN}

Los resultados indican que vivir en una ciudad que se considera segura aumenta el bienestar subjetivo. Un aumento de una unidad en la percepción de seguridad en la ciudad incrementa en un 7,3\% la probabilidad de estar satisfecho con la vida, y disminuye en un 3,4\% 
la probabilidad de sentirse insatisfecho. Por su parte, un aumento de una unidad en la percepción de seguridad en el barrio aumenta en un $1,7 \%$ la probabilidad de estar satisfecho con la vida, y disminuye en un $0,8 \%$ la probabilidad de sentirse insatisfecho. Las variables de seguridad tienen una fuerte relación positiva con la satisfacción con la vida, y la percepción de seguridad en la ciudad tiene mayor impacto que la percepción de seguridad en los barrios.

En cambio, haber sido víctima de crimen disminuye en un 1,1\% la probabilidad de sentirse satisfecho con la vida, y aumenta en un $0,5 \%$ la probabilidad de sentirse insatisfecho.

\section{SATISFACCIÓN CON EL GOBIERNO}

Como medidas de control se incluyeron variables de satisfacción relacionadas con aspectos del gobierno. Todas las variables resultaron significativas y relacionadas positivamente con la satisfacción con la vida, salvo la favorabilidad del concejo. Un aumento en la favorabilidad del alcalde, de la satisfacción con la gestión del equipo de gobierno y con la inversión de recursos públicos, aumenta la probabilidad de estar satisfecho con la vida en un $0,6 \%$, un $1,2 \%$ y un $3,3 \%$ respectivamente. A su vez, un incremento en los niveles de cumplimiento de la ley mejora en un 1,6\% la satisfacción con la vida, y sentir que en la ciudad se garantiza el derecho a la salud aumenta en un $2,4 \%$ la satisfacción con la vida. Dentro de este componente, la variable de mayor impacto es la satisfacción con la inversión de recursos públicos. Esto sugiere que si las administraciones municipales y distritales tienen unas finanzas adecuadas, la ciudadanía apoyará las inversiones que mejoren las condiciones de vida en barrios y ciudades. La eficiencia en la gestión pública puede mejorar la calidad de vida objetiva y modificar la percepción de los ciudadanos acerca de si su ciudad es un buen lugar para vivir (Jiménez, 2015).

\section{EQUIPAMIENTO DEL BARRIO}

Las características físicas, sociales y económicas de los barrios contribuyen significativamente al sentimiento de satisfacción de la comunidad en general (Sirgy y Cornwell, 2002). Los resultados muestran una relación positiva y significativa entre el equipamiento del barrio y la satisfacción con la vida. Si aumenta la satisfacción con las vías, el alumbrado público, los andenes y separadores de las calles, la probabilidad de estar satisfecho con la vida aumenta en un $0,6 \%$, un $1,5 \%$ y un $1,1 \%$ respectivamente. 
El índice de satisfacción con los servicios públicos domiciliarios, construido específicamente para este trabajo, mostró que su incremento aumenta en un 13,5\% la probabilidad de que las personas se sientan satisfechas con la vida.

\section{SOCIOECONÓMICAS}

Las personas de mayor edad tienden a estar más satisfechas con la vida; el aumento de un año de edad incrementa la satisfacción en un 1\%. La variable de género no juega un papel importante en la satisfacción con la vida, un resultado contrario a los que obtuvieron Powdthavee (2005), Davies y Hinks (2010) y Sulemana (2015), quienes encontraron que ser víctima de un crimen tiene un efecto negativo diferencial entre hombres y mujeres. Por último, la pobreza subjetiva refleja una baja satisfacción con la vida. Sentirse pobre disminuye en un 3,8\% la probabilidad de estar satisfecho con la vida.

En síntesis, las variables de mayor impacto fueron, en su orden: satisfacción con los servicios públicos, sensación de seguridad en la ciudad, haber sido víctima de crimen y pobreza subjetiva. Y tomando como referencia a Barranquilla, Bogotá, Cali, Cartagena y Medellín tienen en promedio un menor nivel de satisfacción con la vida; y Manizales y Valledupar un mayor nivel; mientras que es estadísticamente igual al de Barranquilla. Y, como ya se dijo, en el periodo de estudio la satisfacción con la vida disminuyó considerando el promedio de todas las ciudades.

\section{RESULTADO DE LA PRUEBA DE ROBUSTEZ}

Como ya se explicó, para verificar la exogeneidad de la victimización, se llevó a cabo un PSM y se comprobó su sensibilidad a cambios en los supuestos. El efecto es estadísticamente significativo para $\Gamma=1$ y aún más significativo para valores crecientes de $\Gamma$ en los que se pudo haber estimado de modo incorrecto el efecto verdadero del tratamiento.

Cuadro 3

Análisis de sensibilidad del PSM para sesgo oculto con límites de Rosenbaum

\begin{tabular}{lccc}
\hline \multirow{2}{*}{ Gamma $\Gamma$} & \multicolumn{3}{c}{ Valor $\mathrm{p}$} \\
\cline { 2 - 4 } & Kernel & Vecino más cercano & Radial \\
\hline 1 & 0,00000 & 0,00000 & 0,00000 \\
1,1 & 0,00000 & 0,00000 & 0,00000 \\
1,2 & 0,00000 & 0,00001 & 0,00000 \\
1,3 & 0,00000 & 0,00049 & 0,00000 \\
1,4 & 0,00000 & 0,00869 & 0,00004 \\
\hline
\end{tabular}

Fuente: elaboración propia. 
Esto implica que incluso si hay un alto nivel de heterogeneidad no observada, el efecto de ser víctima de la delincuencia en la satisfacción con la vida aún es fuerte, como indican los valores p. Por ejemplo, si no se pude dar cuenta de una variable no observada asociada a un aumento del $50 \%$ en la probabilidad de victimización (es decir, $\Gamma=1,5)$ y si esa variable tiene una fuerte relación con la satisfacción con la vida, el nivel de significación del coeficiente de victimización $(\mathrm{p}=3.90 \mathrm{E}-05)$, después de ajustar por la variable no observada, sigue siendo alto. Estos resultados implican que el sesgo oculto no impide que haya una relación causal entre victimización y seguridad y satisfacción con la vida, de modo que los resultados de este trabajo no tienen los problemas mencionados por Medina et al. (2010).

\section{CONCLUSIONES}

En este trabajo se estimó un modelo probabilístico ordenado para conocer el cambio en la probabilidad de tener una mejor satisfacción con la vida, considerando victimización por crimen, la percepción de seguridad y otras variables de control. Las principales conclusiones son las siguientes:

1. Haber sido víctima reciente de un delito disminuye la probabilidad de estar satisfecho con la vida, es decir, tiene un efecto negativo sobre el bienestar subjetivo de las personas. Si el objetivo de las políticas públicas es mejorar la calidad de vida, además de otros factores se debe controlar la criminalidad y, con ello, la probabilidad de ser víctima de algún delito. Los resultados muestran que el efecto de la criminalidad en el bienestar de los ciudadanos es alto, de modo que el gobierno debe invertir más recursos y tomar medidas más efectivas para reducir la delincuencia.

2. La percepción de seguridad en las ciudades y en los barrios tiene un fuerte efecto positivo en la satisfacción con la vida, aunque es mayor el de la percepción de seguridad en la ciudad que el de la percepción de seguridad en los barrios. En las ciudades colombianas aún existen altas tasas de criminalidad, de modo que hay un alto número de víctimas potenciales.

3. Estar satisfecho con la gestión del gobierno tiene un gran efecto positivo en la probabilidad de que estar satisfecho con la vida, de modo que la transparencia de los niveles de gobierno, la adecuada inversión de recursos públicos, la garantía del derecho a la salud y el cumplimiento de la ley mejoran el bienestar subjetivo de la población. La relación entre satisfacción con la vida y percepción de la gestión 
de los gobiernos locales no ha sido abordada por la literatura reciente; este trabajo es un aporte a la discusión de los factores que determinan el bienestar subjetivo.

4. Las características físicas de los barrios contribuyen significativamente a mejorar el sentimiento de satisfacción con la vida en la comunidad, en especial la buena prestación de servicios públicos domiciliarios.

5. A medida que aumenta la edad mejora la satisfacción con la vida. El género no juega un papel importante, el grado de satisfacción es similar para hombres y mujeres. En cambio, la pobreza subjetiva tiene una fuerte relación con la satisfacción con la vida.

Este trabajo tiene algunas limitaciones empíricas que se espera superar en estudios posteriores. Si bien aborda la endogeneidad y los efectos fijos no observados con pruebas de robustez, estudios futuros podrían dar mayor solidez a los resultados usando datos de panel, experimentos aleatorios o variables instrumentales. Otra limitación es que a las personas encuestadas solo se les preguntó acerca de la victimización en los doce meses anteriores, y no es posible probar si los efectos de la victimización por crimen en la satisfacción con la vida son persistentes o transitorios. Este problema se podría resolver con datos de panel.

\section{REFERENCIAS BIBLIOGRÁFICAS}

Akaike, H. (1974). A new look at the statistical model identification. IEEE Transactions on Automatic Control, 19(6), 716-723.

Amaya, L., Espinosa, A. et al. (2011). Relaciones entre el temor al delito y el autoritarismo de derecha en estudiantes universitarios de LimaPerú. Boletin de Psicología, 103, 7-28.

Anguas, A. (2000). El bienestar subjetivo en la cultura mexicana. Tesis doctoral. Universidad Nacional Autónoma de México, México DF.

Anguas, A. (2001). Identificación y validación del significado del bienestar subjetivo en México: fundamentos para el desarrollo de un instrumento de medición. Journal of Psychology, 35(1), 163-183.

Bissler, D. (2003). Fear of crime and social networks: A community study of two local public housing complexes. Ph.D. dissertation. North Carolina State University.

Blanchflower, D. y Oswald, A. (2004). Well-being over time in Britain and the USA. Journal of Public Economics, 88(7-8), 1359-1386.

Blau, J. R. y Blau, P. M. (1982). The cost of inequality: Metropolitan structure and violent crime. American Sociological Review, 47(1), 114129.

Britt, C. L. (2001). Health consequences of criminal victimization. International Review of Victimology, 8(1), 63-73. 
Cheng, Z. y Smyth, R. (2015). Crime victimization, neighborhood safety and happiness in China. Economic Modelling, 51, 424-435.

Clark, A. y Oswald, A. (1994). Unhappiness and unemployment. Economic Journal, 104(424), 648-659.

Cohen, L. E. y Cantor, D. (1981). Residential burglary in the United States: Life-style and demographic factors associated with the probability of victimization. Journal of Research in Crime and Delinquency, 18(1), 113-127.

Cohen, L. E. y Felson, M. (1979). Social change and crime rate trends: A routine activity approach. American Sociological Review, 44, 588-608.

Cohen, M. A. (2008). The effect of crime on life satisfaction. Journal of Legal Studies, 37(S2), S325-S353.

Cornaglia, F., Feldman, N. E. et al. (2014). Crime and mental well-being. Journal of Human Resources, 49(1), 110-140.

Cronbach, L. J. (1951). Coefficient alpha and the internal structure of tests. Psychometrika, 16(3), 297-334.

Cruz, J. M. (1999). La victimización por violencia urbana: niveles y factores asociados en ciudades de América Latina y España. Revista Panamericana de Salud Pública, 5(4/5), 259-267.

Davies, S. y T Hinks. (2010). Crime and happiness amongst heads of households in Malawi. Journal of Happiness Studies, 11(4), 457-476.

Denkers, A. J. y Winkel, F. W. (1998). Crime victims' well-being and fear in a prospective and longitudinal study. International Review of Victimology, 5(2), 141-162.

Di Tella, R., MacCulloch, R. et al. (2008). Happiness and beliefs in criminal environments RES. Washington DC: BID working paper 4605.

Díaz, A., Formisano, M. et al. (2007). Conflicto, crimen violento y actividad en Colombia: un análisis espacial. En F. Sánchez, (ed.), Las cuentas de la violencia (pp. 461-485). Bogotá: CEDE.

Díaz, G. (2001). E1 bienestar subjetivo. Actualidad y perspectivas. Revista Cubana de Medicina General Integral, 17(6), 572-579.

Díaz. I. C., Ovalle, A. et al. (2001). Actitudes sociales hacia la delincuencia: su relación con la formación académica, el autoritarismo, la victimización $y$ el temor al delito. Tesis de grado. Universidad Nacional de Colombia, Bogotá.

Diener, E., Emmons, R. et al. (1985). The satisfaction with life scale. Journal of Personality Assessment, 49(1), 71-75.

Diener, E., Suh, E. M. et al. (1999). Subjective well-being: Three decades of progress. Psychological Bulletin, 125(2), 276-302.

Diener, E. y González, E. (2011). The validity of life satisfaction measures. Social Indicator Network News, 108, 1-5.

Easterlin, R. (1974). Does economic growth improve the human lot? En P. David y M. Reder (eds.), Nations and households in economic growth: Essays in honour of Moses Abramowitz (pp. 89-125). Nueva York: Academic Press.

Easterlin, R. (2001) Income and happiness: Towards a unified theory. Economic Journal, 111(473), 465-484.

Fierro, A. (2000). Sobre la vida feliz. Málaga: Aljibe. 
Föhrig, A. (2006). Introducción. En J. Varat y A. Garland (eds.), Participación ciudadana y percepción de inseguridad en América Latina (pp. 1-3). Washington DC: Woodrow Wilson International Center for Scholars.

Franzini, L., Caughy, M. et al. (2008). Perceptions of disorder: Contributions of neighborhood characteristics to subjective perceptions of disorder. Journal of Environmental Psychology, 28(1), 83-93.

Garofalo, J. (1973). The fear of crime: Causes and consequences. Journal of Criminal Law and Criminology, 72(2), 839-857.

Garofalo, J. (1979). Victimization and the fear of crime. Journal of Research in Crime and Delinquency, 16(1), 80-97.

Gaviria, A., Medina, C. et al. (2010). The cost of avoiding crime: The case of Bogotá. En R. di Tella, S. Edwards, et al. (eds.), The economics of crime: Lessons for and from Latin America (pp. 175-204). Chicago: NBER y University of Chicago Press.

Giovannini, E., Nardo, M. et al. (2005). Handbook on constructing composite indicators: methodology and user guide. París: OECD Statistics working paper 2.

Graham, C. y Pettinato, S. (2002). Happiness and hardship: opportunity and insecurity in New market economies. Washington DC: Brookings Institution Press.

Hanslmaier, M. (2013). Crime, fear and subjective well-being: How victimization and street crime affect fear and life satisfaction. European Journal of Criminology, 10(5), 515-533.

Hanson, R., Sawyer, G. et al. (2010). The impact of crime victimization on quality of life. Journal of Traumatic Stress, 23(2), 189-197.

Hunter, A. (1978). Symbols of incivility: Social disorder and fear of crime in urban neighborhoods. Reactions to crime project. Washington DC: working paper M-46A

Jiménez, E. U. (1985). Análisis de series temporales modelos Arima. Málaga: Thomson-Paraninfo.

Jiménez, O. (2015). Informe de calidad de vida, comparando 14 ciudades 2011-2013. Red Ciudades Cómo vamos. Boletín 6, 1-126.

Kahneman, D. (1989). Objective happiness. En D. Kahneman, E. Diener, et al. (eds.), Well-being: The foundations of hedonic psychology (pp. 3-25). Nueva York: Russell Sage.

Kessler, G. (2009). El sentimiento de inseguridad: sociología del temor al delito. Buenos Aires: Siglo Veintiuno.

Koskela, H. (2011). El desafío del temor-delito y temor al delito como problemas sociales urbanos. Brazilian Geographical Journal: Geosciences and Humanities research medium, 2(2), 274-285.

Koss, M. P., Koss, P. G. et al. (1991). Deleterious effects of criminal victimization on women's health and medical utilization. Archives of internal medicine, 151(2), 342-347.

Koss, M. P., Woodruff, W. J. et al. (1990). Relation of criminal victimization to health perceptions among women medical patients. Journal of Consulting and Clinical Psychology, 58(2), 147.

Kullback, S. y Leibler, R. A. (1951). On information and sufficiency. Annals of Mathematical Statistics, 22(1), 79-86. 
Kuroki, M. (2013). Crime victimization and subjective well-being: Evidence from happiness data. Journal of Happiness Studies, 14(3), 783-794.

Latkin, C. A. y Curry, A. D. (2003). Stressful neighborhoods and depression: A prospective study of the impact of neighborhood disorder. Journal Health and Social Behavior, 44(1), 34-44.

Latkin, C. A., German, D. et al. (2009). Individual-level influences on perceptions of neighborhood disorder: A multilevel analysis. Journal of Community Psychology, 37(1), 122-133.

Lavrakas, P. y Lewis, D. (1980). The conceptualization and measurement of citizens' crime prevention behaviors. Journal of Research in Crime and Delinquency, 17(2), 254-272.

Layard, R. (2005). Happiness: Lessons from a new science. Nueva York: Penguin Books.

Lelkes, O. (2006). Knowing what is good for you: Empirical analysis of personal preferences and the 'Objective Good'. Journal of Socioeconomics, 35(2), 285-307.

Long, S. J. y Freese, J. (2006). Regression models for categorical dependent variable using stata 2 nd ed. College Station. TX: Stata press.

Lorenc, T., Clayton, S. et al. (2012). Crime, fear of crime, environment, and mental health and wellbeing: mapping review of theories and causal pathways. Health E place, 18(4), 757-765.

Medina, C. y Tamayo, A. (2012). An assessment of how urban crime and victimization affects life satisfaction. En D. Webb y E. Wills-H. (eds.), Subjective well-being and security (pp. 91-147), Berlin: Springer.

Medina, C., Morales, L. et al. (2010). Quality of life in urban neighbourhoods in Bogota and Medellin, Colombia. En E. Lora, A. Powell et al. (eds.), The quality of life in Latin American cities: Markets and perceptions (pp. 117-160). Washington DC: Inter-American Development Bank and the World Bank.

Medina, J. (2003). Inseguridad ciudadana, temor al delito y policía en España. Revista de Psicología, 6(2), 143-150.

Messner, S. F. (1982). Poverty, inequality, and the urban homicide rate: Some unexpected findings. Criminology, 20(1), 103-114.

Messner, S. F. y Tardiff, K. (1986). Economic inequality and levels of homicide: An analysis of urban neighborhoods. Criminology, 24(2), 297-316.

Michalos, A. C. y Zumbo, B. D. (2000). Criminal victimization and the quality of life. Social Indicators Research, 50(3), 245-295.

Min, H. (2013). Ordered logit regression modeling of the self-rated health in Hawai' $i$, with comparisons to the OLS Model. Journal of Modern Applied Statistical Methods, 12(2), 371-380.

Moller, V. (2005). Resilient or resigned? Criminal victimization and quality of life in South Africa. Social Indicators Research, 72(3), 263-317.

Moore, S. C. (2006). The value of reducing fear: An analysis using the European Social Survey. Applied Economics, 38(1), 115-117.

Moore, S. y Shepherd, J. P. (2006). The cost of fear: shadow pricing the intangible costs of crime. Applied Economics, 38(3), 293-300.

Moscote, O. y Rincón, W. (2012). Modelo logit y probit: un caso de aplicación. Comunicaciones en Estadística, 5(2), 123-133. 
Moutinho, L., Peel, J. et al. (1998). Estimating consumer satisfaction: OLS versus ordered probability models. International Journal of Commerce and Management, 8(2), 75-93.

Pavot, W., Diener, E. et al. (1991). Further validation of the satisfaction with life scale: Evidence for the cross-method convergence of wellbeing. Social Indicators Research, 28, 1-20.

Pavot, W. y Diener, E. (2008). The satisfaction with life scale and the emerging construct of life satisfaction. Journal of Positive Psychology, 3(2), 137-152.

Peña, J. L. (2005). Temor al crimen en Bogotá: un estudio empirico. Tesis de grado. Universidad Nacional de Colombia, Bogotá.

Pérez-E., N. (2013). Variables predictivas de la satisfacción con la vida en estudiantes universitarios. En C. Cardona, et al. (eds.), Investigación e innovación educativa al servicio de Instituciones y comunidades globales, plurales $y$ diversas (pp. 1548-1552). Madrid: AIDIPE.

Powdthavee, N. (2005). Unhappiness and crime: Evidence from South Africa. Economica, 72(3), 531-547.

Powdthavee, N. (2009). I can't smile without you: Spousal correlation in life satisfaction. Journal of Economic Psychology, 30(4), 675-689.

Propper, C., Burgess, S. et al. (2007). The impact of neighbourhood on the income and mental health of British social renters. Urban Studies, 44(2), 393-415.

Riger, G. (1978). Women's fear of crime: From blaming to restricting the victim. Victimology, 3, 274-284.

Rojas, M. (2006). Life satisfaction and satisfaction in domains of life: Is it a simple relationship? Journal of Happiness Studies, 7(4), 467-497.

Romero, D. (2014). Insecurity or perceptions of insecurity? Urban crime and dissatisfaction with life: Evidence from the case of Bogota. De Gruyter, 20(1), 169-208.

Rosenbaum, P. R. (2002). Observational studies. Nueva York: Springer.

Ross, C. E. Reynolds, J. R. et al. (2000). The contingent meaning of neighborhood stability for residents' psychological well-being. American Sociological Review, 65(4), 581-597.

Ruiz, J. I. (2007). Cultura ciudadana, temor al crimen y victimización: un análisis de sus interrelaciones desde la perspectiva del tejido social. Acta Colombiana de Psicología, 10(1), 65-74.

Russo, S. y Roccato, M. (2010). How long does victimization foster fear of crime? A longitudinal study. Journal of Community Psychology, 38(8), 960-974.

Sampson, R. J. y Raudenbush, S. W. (2004). Seeing disorder: Neighborhood stigma and the social construction of 'broken windows'. Social Psychology Quarterly, 67(4), 319-342.

San Juan, C., Vergara, A. et al. (2005). Propiedades psicométricas de un cuestionario para la evaluación de la calidad de vida urbana y el temor al delito. Revista Española de Investigación Criminológica, 3, 2-13.

Schimmack, U. y Oishi, S. (2005). The influence of chronically accessible and temporarily accessible information on life satisfaction judgments. Journal of Personality and Social Psychology, 89(3), 395-406. 
Schuschny, A. y Soto, H. (2009). Guía metodológica. Diseño de indicadores compuestos de desarrollo sostenible. Documento de proyecto 255. Santiago de Chile: Cepal.

Schwarz, N. y Strack, F. (1999). Reports of subjective well-being: Judgmental processes and their methodological implications. En D. Kahneman, E. Diener et al. (eds.), Well-being: The foundations of hedonic psychology (pp. 61-84). Nueva York: Russell Sage.

Sirgy, M. J. y Cornwell, T. (2002). How neighborhood features affect quality of life. Social Indicators Research, 59(1), 79-114.

Skogan, W. G. y Maxfield, M. G. (1981). Coping with crime: Individual and neighborhood. Washington DC: Sage.

Soares, R. y Naritomi, J. (2010). Understanding high crime rates in Latin America: The role of social and policy factors. En R. di Tella, S. Edwards, et al. (eds.), The economics of crime: Lessons for and from Latin America (pp. 19-55). Chicago: NBER-University of Chicago Press.

Stafford, M., Chandoia, T. et al. (2007). Association between fear of crime and mental health and physical functioning. American Journal of Public Health, 97(11), 2076-2081.

Staubli, S., Killias, M. et al. (2014). Happiness and victimization: An empirical study for Switzerland. European Journal of Criminology, 11(1), 57-72.

Sulemana, I. (2015). The effect of fear of crime and crime victimization on subjective well-being in Africa. Social Indicators Research, 121(3), 849-872.

Takane, Y. (1987). Introduction to special section. Psychometrika, 52(3), 315-316.

Tittle, C. R. y Villemez, W. J. (1977). Social class and criminality. Social Forces, 56(2), 474-502.

Tittle, C. R. Villemez, W. J. et al. (1978). The myth of social class and criminality: An empirical assessment of the empirical evidence. American Sociological Review, 43(5), 643-656.

Vilalta, C. (2011). Fear of crime in public transport: Research in Mexico City. Crime Prevention E Community Safety, 13(3), 171-186.

Visser, M., Scholte, M. et al. (2013). Fear of crime and feelings of unsafety in European countries: Macro and micro explanations in cross-national perspective. Sociological Quarterly, 54(2), 278-301.

Vozmediano, L., San Juan, C. et al. (2008). Problemas de medición del temor al delito: Algunas respuestas teóricas y técnicas. Revista Electrónica de Ciencia Penal y Criminología, 10(7), 1-17.

Ward, R. A., LaGory, M. et al. (1986). Fear of crime among the elderly as person/environment interaction. Sociological Quarterly, 27(3), 327-341.

Zamarrón, M. D. (2006). E1 bienestar subjetivo en la vejez. Lecciones de gerontología. Portal Mayores, 52, 4-18. 